\title{
On the Impact of Channel Cross-Correlations in High-Sensitivity Receivers for Galileo E1 OS and GPS L1C Signals
}

\author{
Davide Margaria, ${ }^{1}$ Beatrice Motella, ${ }^{1}$ and Fabio Dovis ${ }^{2}$ \\ ${ }^{1}$ Istituto Superiore Mario Boella, Via P.C. Boggio 61, 10138 Torino, Italy \\ ${ }^{2}$ Department of Electronics and Telecommunications, Politecnico di Torino, Corso Duca degli Abruzzi 24, 10129 Torino, Italy
}

Correspondence should be addressed to Davide Margaria, margaria@ismb.it

Received 31 December 2011; Accepted 5 April 2012

Academic Editor: Shaojun Feng

Copyright (C) 2012 Davide Margaria et al. This is an open access article distributed under the Creative Commons Attribution License, which permits unrestricted use, distribution, and reproduction in any medium, provided the original work is properly cited.

\begin{abstract}
One of the most promising features of the modernized global navigation satellite systems signals is the presence of pilot channels that, being data-transition free, allow for increasing the coherent integration time of the receivers. Generally speaking, the increased integration time allows to better average the thermal noise component, thus improving the postcorrelation SNR of the receiver in the acquisition phase. On the other hand, for a standalone receiver which is not aided or assisted, the acquisition architecture requires that only the pilot channel is processed, at least during the first steps of the procedure. The aim of this paper is to present a detailed investigation on the impact of the code cross-correlation properties in the reception of Galileo E1 Open Service and GPS L1C civil signals. Analytical and simulation results demonstrate that the S-curve of the code synchronization loop can be affected by a bias around the lock point. This effect depends on the code cross-correlation properties and on the receiver setup. Furthermore, in these cases, the sensitivity of the receiver to other error sources might increase, and the paper shows how in presence of an interfering signal the pseudorange bias can be magnified and lead to relevant performance degradation.
\end{abstract}

\section{Introduction}

In the context of Global Navigation Satellite Systems (GNSS) receivers, the interest on the new modulations that will be used for the modernized GPS L1C and Galileo E1 Open Service (OS) civil signals grew rapidly in past years. The definition of new signals structure results from an agreement between the European Commission and Unites States of America. A common Multiplexed Binary Offset Carrier Modulation (MBOC) signal baseline has been adopted, with the aim of assuring the compatibility and interoperability between GPS and Galileo systems [1]. For the GPS L1C signal, USA has chosen the Time Multiplexed BOC (TMBOC) solution that multiplexes a $\operatorname{BOC}(1,1)$ with a $\operatorname{BOC}(6,1)$ in time domain [2], while the composite $\mathrm{BOC}$ (CBOC) is the implementation selected for the Galileo E1 OS Signal In Space (SIS) [3].

One of the main features of the modernized civil and open access signals is the presence of the pilot channels. Pilot channel has been introduced to allow the receivers to perform coherent integration over a long time, without facing the issue of unpredictable data transitions. As a consequence, the receiver is able to acquire satellite signals at lower SNR than the nominal value. In order to deal with such a need in current GPS receiver, assistance data have been defined and standardized [4] in order to overcome the issues induced by the presence of data transition, which arms the entire correlation process. When dealing with standalone receivers, the presence of the pilot channel is of utmost importance since it allows to increase the integration time but avoiding the data wipe-off. In such a case, the correlation is performed with a local version of the code of the pilot channel, and the correlation value becomes the decision metrics for the detection of the satellite.

In this paper, the distortion of the discrimination function (S-curve) due to codes cross-correlation properties is assessed, considering the features of the modulation schemes adopted in Galileo E1 OS and GPS L1C civil signals and also investigating different receiver configurations (reception of data/pilot channels, variable correlators spacing). 
This article is based on the preliminary results presented in [5]. A more detailed analysis has been carried out in order to assess and compare the auto- and cross-correlation properties of different families of codes and their impact on the receiver performance.

After this introduction, the paper is organized as follows: Section 2 discusses the features of Galileo E1 OS and GPS L1C modulations. Then, the impact of the codes crosscorrelations and the receiver setup on the discrimination function is analysed (Sections 3 and 4). Simulation results are presented in Section 5 in order to show that, receiving a single channel (e.g., the pilot channel), the code cross-correlation distortion on the S-curve can be magnified by an inappropriate choice of the correlator spacing, leading to noticeable worsening in receiver performance when interfering signals are present. Section 6 outlines the conclusions.

\section{Galileo E1 OS and GPS L1C Modulation Features}

The MBOC signal baseline assures more power to the highfrequency spectral components if compared to the baseline $\operatorname{BOC}(1,1)$ and $\operatorname{BPSK}(1)$ modulations. This feature leads to a sharpener code correlation peak allowing to achieve improved tracking performance [6]. At the same time, since an amount of power is allocated to high frequencies, no benefits can be observed for receivers that use narrow frontend bandwidths. The $\operatorname{MBOC}(6,1,1 / 11)$ signal baseline has been recommended to obtain high degree of interoperability with receivers that might use narrowband frontends. In such a case, since more than $90 \%$ of total power $(10 / 11)$ remains available and the filtered $\operatorname{MBOC}(6,1,1 / 11)$ resembles a $\operatorname{BOC}(1,1)$ signal. Moreover, MBOC presents less susceptibility to narrow band interference at the worst case frequency. The TMBOC and CBOC modulations are designed to reduced side-lobe levels in the auto- and crosscorrelation functions.

Both Galileo E1 OS and GPS L1C signals include two channels: the pilot signal, without any data message, that is spread by a ranging (pseudo-random noise-PRN) code; and the data channel, spread by a ranging code and modulated by a data message. At the receiver side, it is possible to consider only one of the two channels in order to exploit peculiar characteristics, as for example, if long integration times have to be used. The Galileo and the GPS signals differ on the modulation formats (CBOC versus TMBOC), on the data/pilot power allocations and on the code properties. Concerning the signals in space, it must be remarked that both the signals will be received with the same total power, that is $-157 \mathrm{dBW}[2,3]$.

In addition, codes belonging to different families (memory codes for Galileo E1 OS signals, Weil codes for GPS L1C) will be used by the two systems in tiered code structures featuring different lengths, as summarized in Table 1.

The differences in terms of code properties, modulation formats (and consequent different spectral occupation), and relative power levels are then expected to lead to different system performance. As an example considering the levels of interference robustness, it has been noticed that receiving only a single channel in case of continuous wave interference [7], better results have been obtained with the GPS L1C pilot channel and with the Galileo E1 data channel. Differences among modulations also depend on the setup of the correlator spacing and, as expected, tend to become negligible narrowing the correlators. More details on CBOC and TMBOC modulations are provided below, considering the correlation properties related to the data and pilot channels coexistence. Code properties will be discussed in the following sections.

2.1. Galileo E1 OS: $C B O C(6,1,1 / 11)$. The main features of the E1 Open Service signal can be summarized as follows:

(i) $50 \%$ power split between data $\left(E 1_{\mathrm{B}}\right)$ and pilot $\left(E 1_{\mathrm{C}}\right)$ channels, for robust data demodulation;

(ii) optimized memory codes;

(iii) use of a tiered code structure including $4 \mathrm{~ms}$ primary and $100 \mathrm{~ms}$ secondary codes on the pilot channel.

Both pilot and data channel components take advantage of the $\operatorname{CBOC}(6,1,1 / 11)$ modulation: each PRN code chip is shaped by a weighted combination of $\operatorname{BOC}(1,1)$ and $\operatorname{BOC}(6,1)$ spreading symbols.

The chip shapes (normalized with unitary power) of the two Galileo E1 OS channels are reported in Figure 1, showing the $\mathrm{CBOC}(6,1,1 / 11)$ modulation. It must be pointed out the different sign in combining the $\operatorname{BOC}(1,1)$ and $\operatorname{BOC}(6,1)$ components between the data channel (denoted as $\mathrm{CBOC}_{+}$ in Figure 1) and the pilot channel (denoted as $\mathrm{CBOC}_{-}$), according to the Galileo OS Interface Control Document [3].

The theoretical autocorrelation functions computed on single chip of data $\left(R_{\mathrm{CBOC}+}\right)$ and pilot channels $\left(R_{\mathrm{CBOC}-}\right)$ are shown in Figure 2 where the data/pilot cross-correlation function $\left(R_{\mathrm{CBOC}_{ \pm}}\right)$is also represented.

In order to obtain unitary autocorrelation peaks, Figure 2 and following correlation plots have been obtained after normalizing the signal amplitudes.

Assuming now to demodulate the received signal (data and pilot channels) by using only the pilot component (local signal replica), the resulting correlation function $R(\tau)$ can be expressed by

$$
R(\tau)=R_{\mathrm{CBOC}_{-}}(\tau)+\alpha \cdot R_{\mathrm{CBOC}_{ \pm}}(\tau),
$$

where $\tau$ is the code delay in chips (omitted hereafter, in order to simplify the notation); $R_{\text {CBOC_}}$ is the (unitary) autocorrelation function of the pilot channel; $R_{\mathrm{CBOC}_{ \pm}}$is the data/pilot cross-correlation function; $\alpha$ is a parameter representing the weight of the cross term, depending on both the data/pilot power splitting and on the code properties (relative amplitudes on the code auto- and cross-correlation functions).

The corresponding discrimination function is depicted in Figure 3, for different $\alpha$, where the typical irregular trend on the slope due to the $\operatorname{BOC}(6,1)$ signal components can be observed. In Figure 3 an incoming signal composed by data and pilot channels is correlated with the pilot signal, 


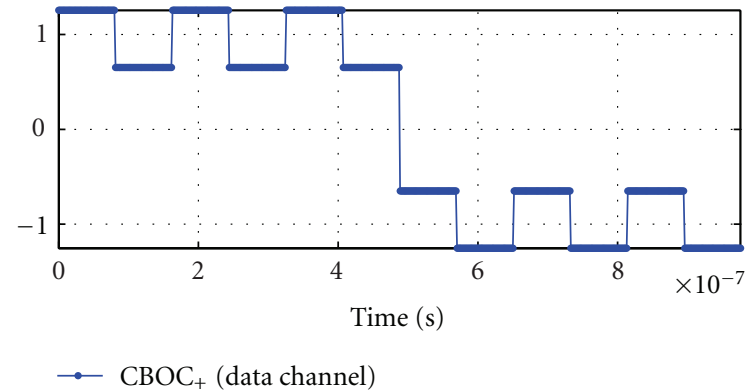

(a)

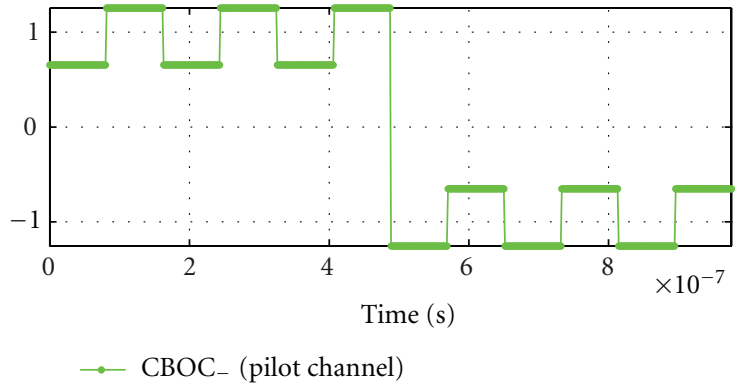

(b)

Figure 1: Chip shape of Galileo E1 OS data channel (in blue) and pilot channel (in green), CBOC modulation $(\mathrm{BOC}(1,1) \pm \mathrm{BOC}(6,1))$.

TABLE 1: Galileo E1 OS and GPS L1C code lengths.

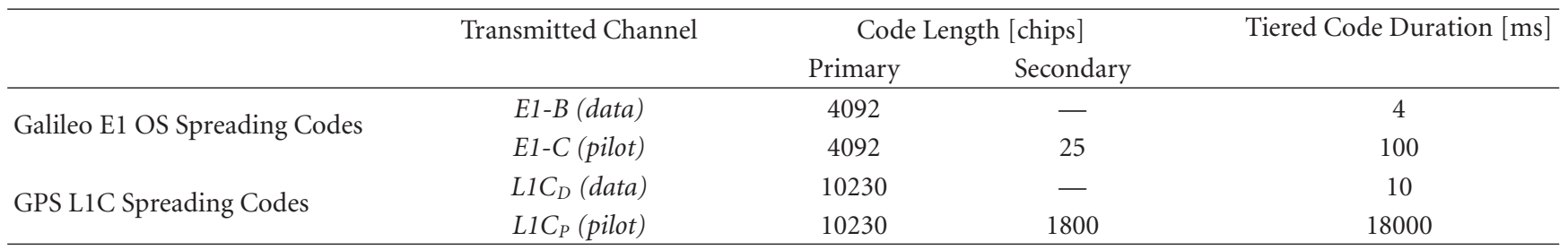

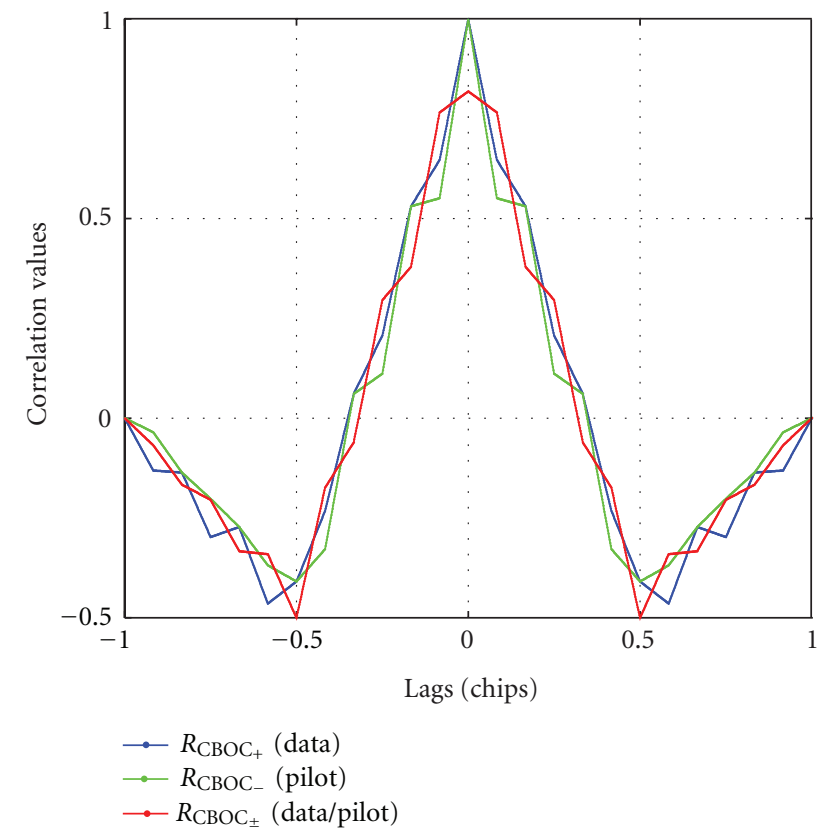

Figure 2: Theoretical CBOC circular correlation functions computed on single chip. Chip shape: $\operatorname{BOC}(1,1) \pm \operatorname{BOC}(6,1)$.

computing a coherent early-late discrimination function (early-late correlator spacing $\Delta$ of 1 chip) for different values of $\alpha$. The variation of $\alpha$ impacts on the S-curve, affecting the slope in the linear region. In addition, it can be noticed that, even if $\alpha$ is different from zero, the discrimination function preserves symmetry and no tracking point biases are introduced.
It is then possible to conclude that the intrinsic $\mathrm{CBOC}$ correlation and discrimination functions always appear symmetrical, regardless to the data/pilot relative power levels. Possible biases around the lock point will be due to other effects (codes cross-correlation impact), as it will be demonstrated in the following sections.

2.2. GPS L1C: $\operatorname{TMBOC}(6,1,4 / 33)$ and $B O C(1,1)$. The $\mathrm{L} 1 \mathrm{C}$ signal, similarly to the Galileo E1 OS, consists of a data $\left(\mathrm{L}_{1 C_{D}}\right)$ channel and a pilot $\left(\mathrm{L}_{1} \mathrm{C}_{\mathrm{P}}\right)$ channel [2]. Its main characteristics can be summarized as follows.

(i) $75 \%$ of power in the pilot component for enhanced signal tracking;

(ii) advanced Weil-based spreading codes;

(iii) use of a long overlay code (18 s) on the pilot channel.

The L1C MBOC implementation modulates the entire data component and 29 of every 33 code chips of the pilot channel with $\operatorname{BOC}(1,1)$, while 4 of every 33 pilot channel chips with a $\operatorname{BOC}(6,1)$ waveform. The code chip shapes for the $\operatorname{BOC}(1,1)$ data channel and the $\operatorname{TMBOC}(6,1,4 / 33)$ pilot channel are shown in Figure 4.

The optimized L1C signal has been designed to assure interoperability with Galileo E1 OS signal. The unequal power split improves the pilot tracking threshold by $1.87 \mathrm{~dB}$ compared with a $50 \%$ power split used in Galileo. It has been shown that a TMBOC pilot usage extends most of the advantages exploited by BOC $(1,1)$ by more than $1 \mathrm{~dB}$ over $\operatorname{BOC}(1,1)$. The L1C modulation has been introduced to enhance the signal robustness in critical environments [8].

The TMBOC implementation assures a MBOC-like spectrum, but implies a slightly different correlation function with respect to the Galileo E1 CBOC case. Notice that, using 


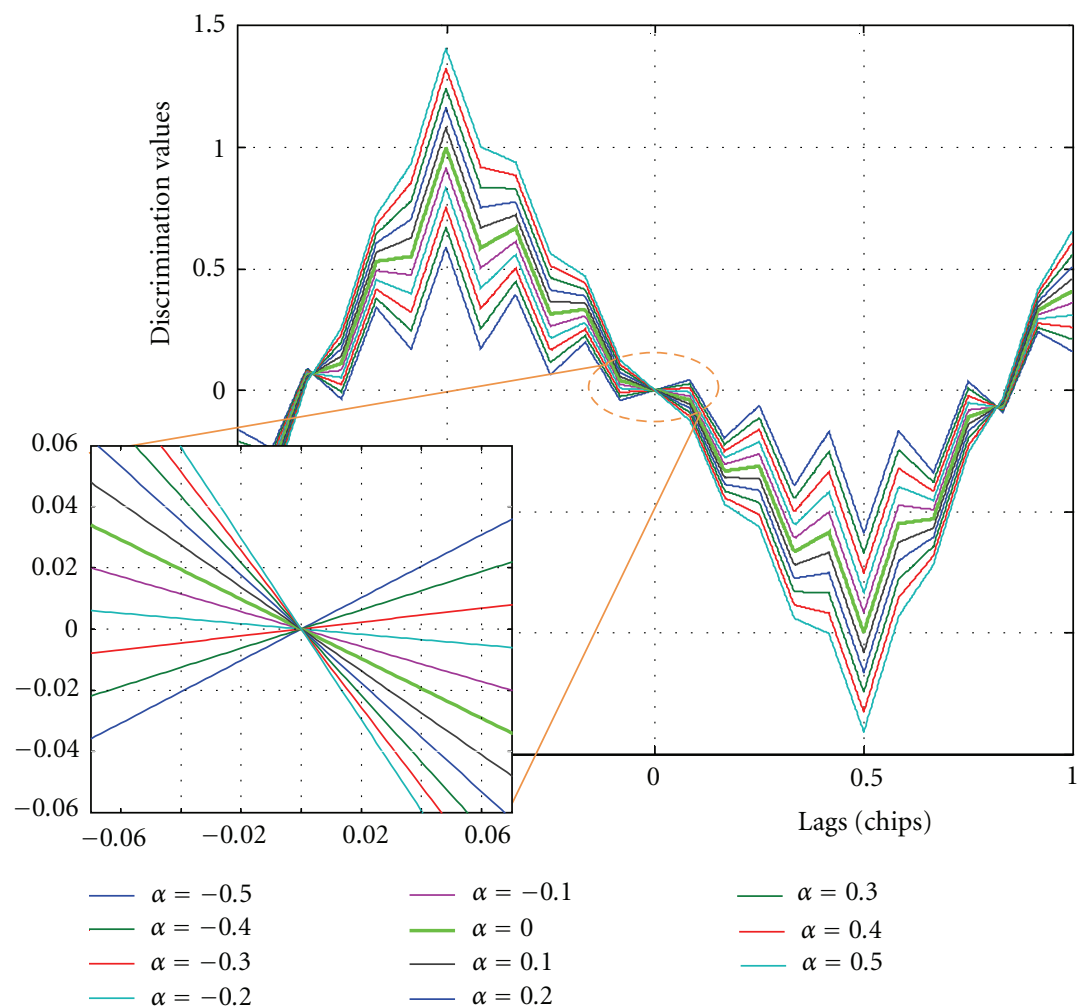

FIGURE 3: Discrimination function (coherent Early-Late, spacing $\Delta=1$ chip) and its zoom, obtained using a single chip of the Galileo E1 OS signal and varying $\alpha$. Received signal: both data and pilot channels, local signal: pilot channel only. PRN codes are neglected.

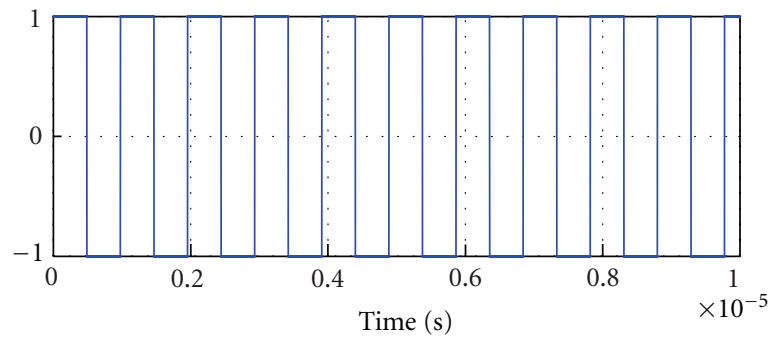

$\rightarrow \operatorname{BOC}(1,1)$ (data channel)

(a)

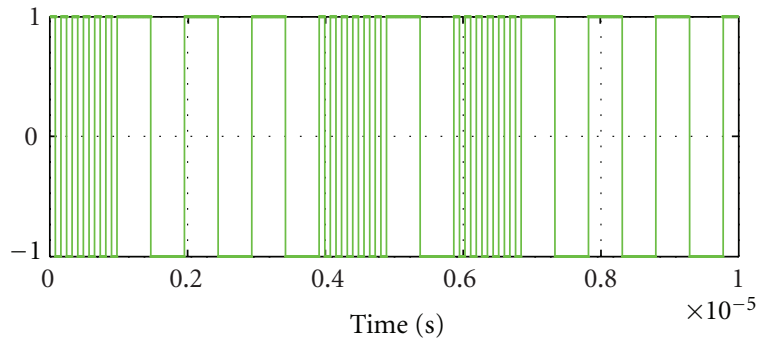

$\therefore \operatorname{TMBOC}(6,1,4 / 33)$ (pilot channel)

(b)

Figure 4: Code chip shape of GPS L1C data channel (in blue) and pilot channel (in green), due to the BOC $(1,1)$ and TMBOC $(6,1,4 / 33)$ modulations. PRN codes are neglected.

this kind of waveform, it is not possible to repeat the previous analysis concerning the theoretical S-curve for a single code chip: the TMBOC is in fact defined over a sequence of 33 chips. In order to obtain meaningful correlation and discrimination functions, it is necessary to use a whole code period.

\section{Codes Cross-Correlation Impact on the S-Curve}

Two are the signal elements that affect the discrimination function shape: the modulation and the code. While the former has already been investigated in the previous section, the impact of the code is the focus of the current one.

Both the Galileo E1 OS and the GPS L1C signals are taken into account, with specific analyses on how different code families can affect the shape of the discrimination function. In detail the memory codes (introduced for Galileo E1 OS) and the Weil codes (specific for GPS L1C) will be discussed.

3.1. Galileo E1 OS: Memory Codes. Memory codes are foreseen for the Galileo E1 OS signal [3]. 4092 chips 4 mslong codes will be implemented as primary codes, in a tiered code structure (see Table 1). 


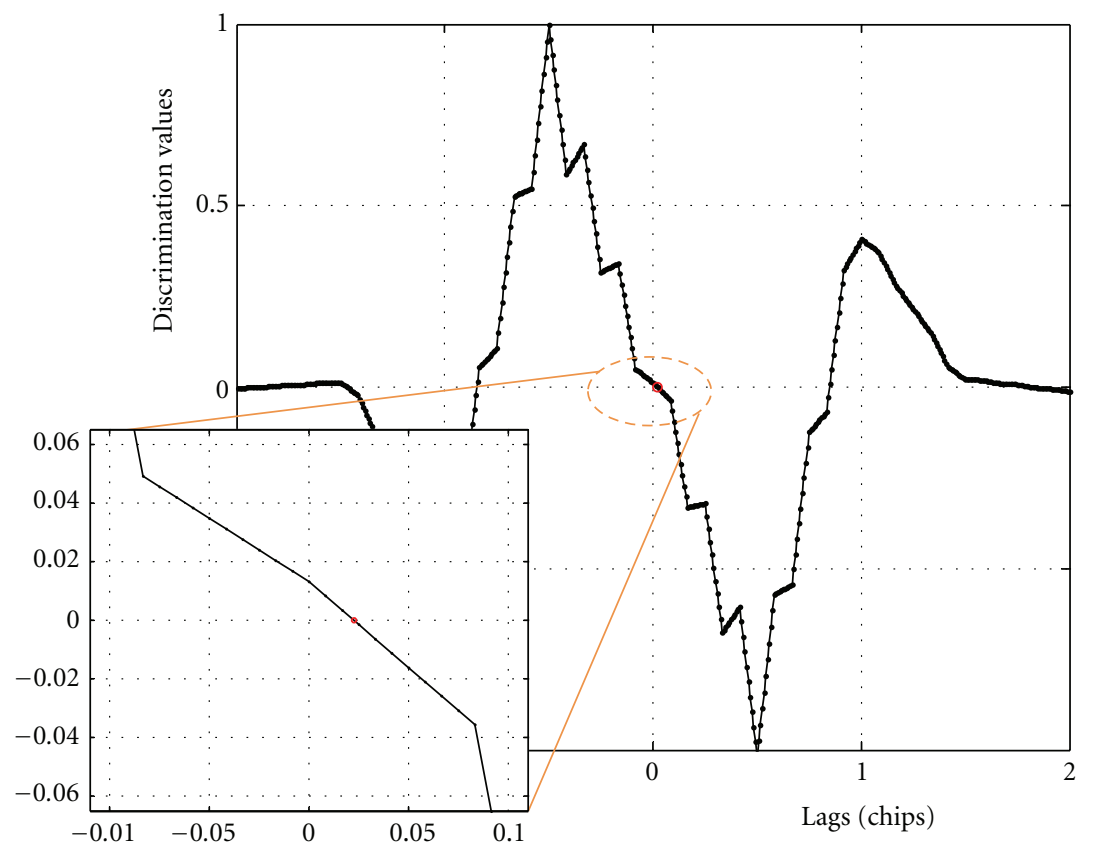

FIGURE 5: Discrimination function (coherent Early-Late, $\Delta=1$ chip) and its zoom, obtained for a Galileo E1 OS signal using memory codes (4 ms primary codes, PRN 1). Received signal: both data and pilot channels, local signal: pilot channel only. The S-curve does not result symmetrical around the zero lag (bias $=6.61 \mathrm{~m})$.

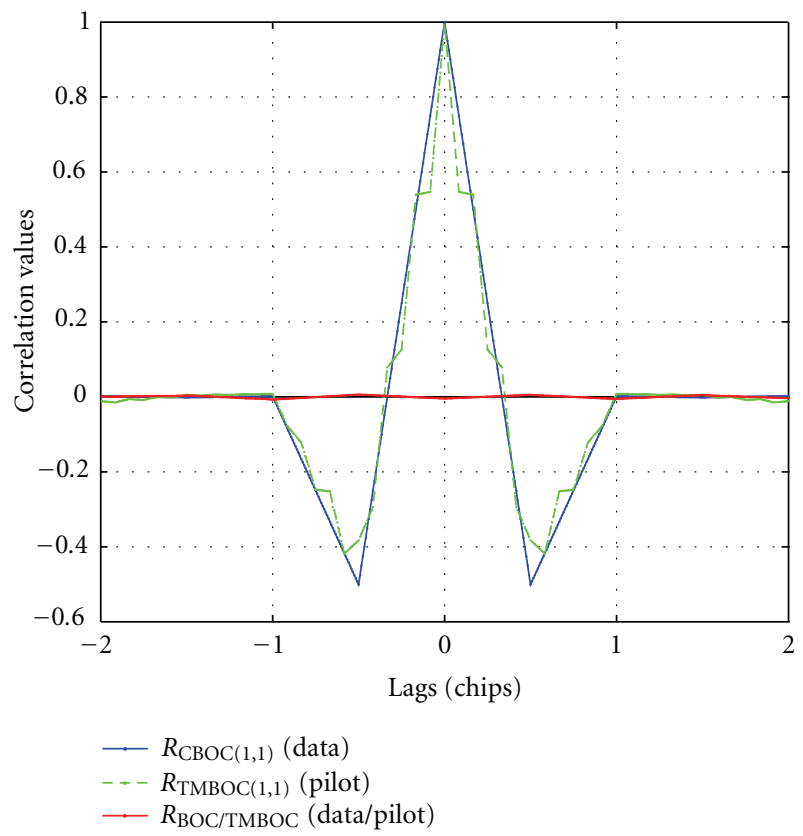

Figure 6: Data (blue line) and pilot (green line) auto-correlation functions, data/pilot cross-correlation function (red line), obtained for a GPS L1C signal using Weil codes ( $10 \mathrm{~ms}$, PRN 1 data and pilot channels).

It must be noted that, in spite of what happens for the Gold codes used by the GPS L1 current signal $[9,10]$, the

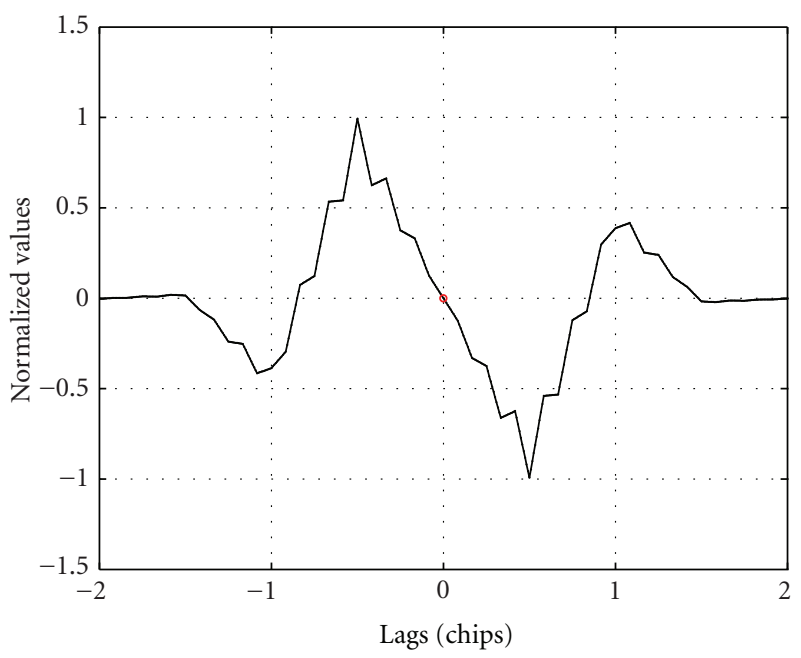

Figure 7: Discrimination function (coherent Early-Late, $\Delta=1$ chip) obtained for a GPS L1C signal using Weil codes (10 ms, PRN 1). Received signal: both data and pilot channels, local signal: pilot channel only. The S-curve does not result symmetrical around the zero lag (bias $=0.09 \mathrm{~m})$.

auto- and cross-correlation functions for memory codes take multiple values.

In addition, as explained in [11], the codes are designed to fulfill special properties, such as low autocorrelation side lobes. This guarantees that the autocorrelation values of every code correlate to zero with a replica of itself, delayed by one chip. 


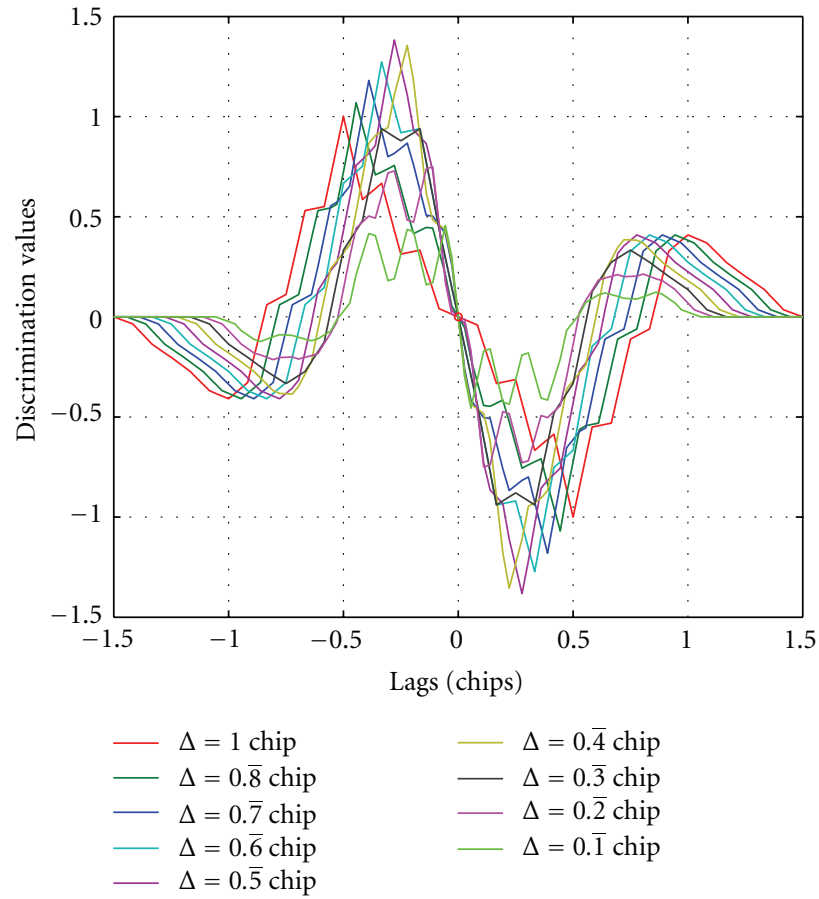

(a)

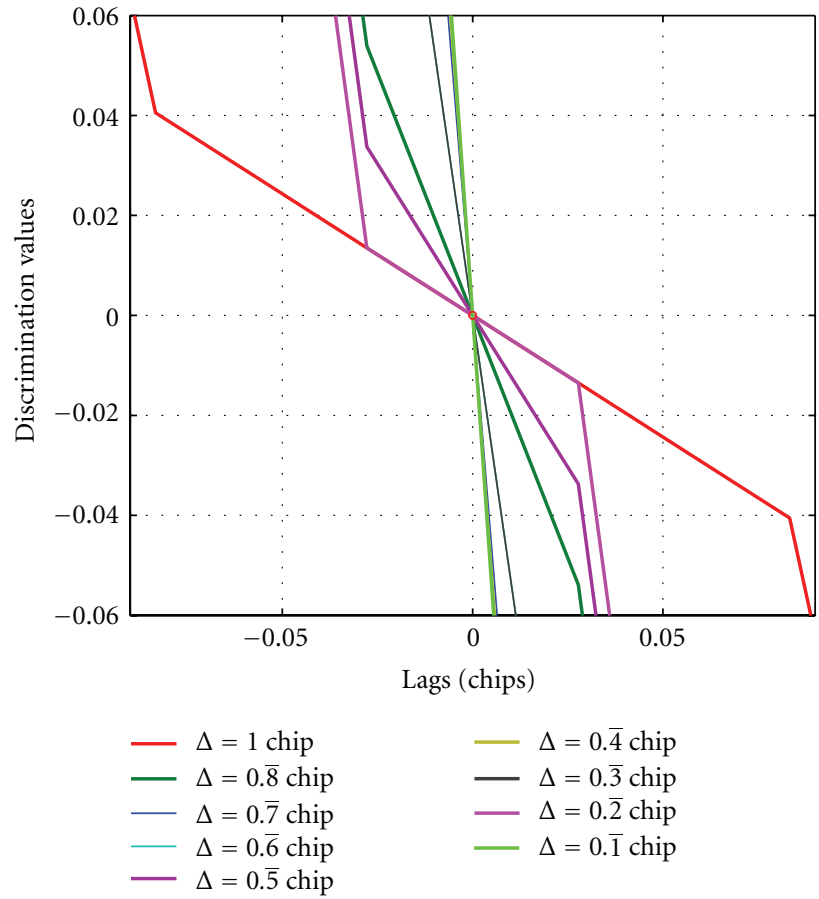

(b)

FIGURE 8: Theoretical coherent Early-Late discrimination function (a) and its zoom (b) varying the correlator spacing ( $\Delta$ ) and considering only the pilot channel of Galileo E1 OS (CBOC modulated chip without code cross-correlation effect, $\alpha=0$ ).

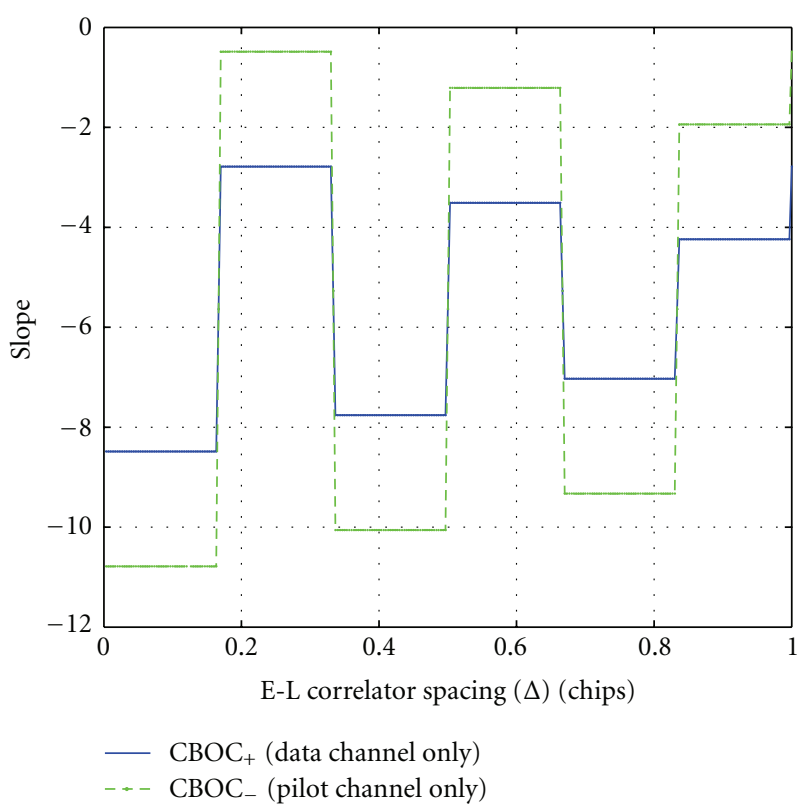

Figure 9: Theoretical S-curve slope varying the correlator spacing, considering both data and pilot channels of a Galileo E1 OS-like signal (CBOC modulated chip without code effect, $\alpha=0$ ).

Proceeding with our analysis, the discrimination function of the Galileo E1 OS CBOC signal (obtained correlating only the pilot channel) might present an asymmetry and a change in the slope around the zero lag. An example is given in Figure 5, where an S-curve for the Galileo E1 OS signal (PRN 1) is depicted, showing a bias of $6.61 \mathrm{~m}$ around the origin.

3.2. GPS L1C. The codes foreseen for the GPS L1C TMBOC signal are Weil codes $[2,12]$. They are based on Legendre sequences of 10230 chips (10 ms).

Also in this case, in spite of what happens for the Gold codes, the auto- and cross-correlation functions of the PRNs $\left(\mathrm{L}_{1} \mathrm{C}_{\mathrm{D}}\right.$ Data channel and $\mathrm{L1C}_{\mathrm{P}}$ Pilot channel) take multiple values but have not been designed to fulfill special properties such as in the case of Galileo memory codes.

The effect of the Weil codes on the S-curve is again the introduction of an asymmetry caused by the crosscorrelation contribution. In this case, due to the code length ( $10 \mathrm{~ms}$, instead of $4 \mathrm{~ms}$ memory codes), the impact on the discrimination function results attenuated. This can be derived by observing Figure 6, where the data and pilot autocorrelation functions (with blue and green lines, resp.) are shown together with the data/pilot cross-correlation function (red line).

The cross-correlation term is not symmetrical, but the impact is this case is reduced. This is due to the fact that Weil codes are longer $(10 \mathrm{~ms})$ and consequently present better correlation properties. In fact, the longer the codes, the smaller the cross-correlation functions they have. This fact causes in a lighter effect on the S-curve asymmetry. The price 


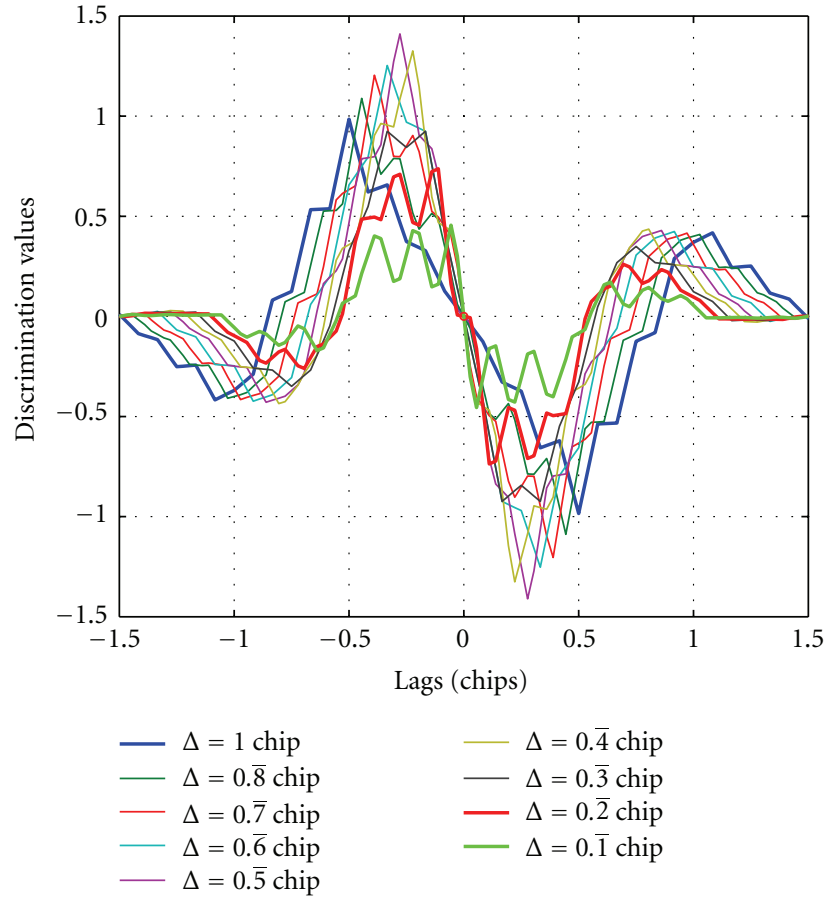

(a)

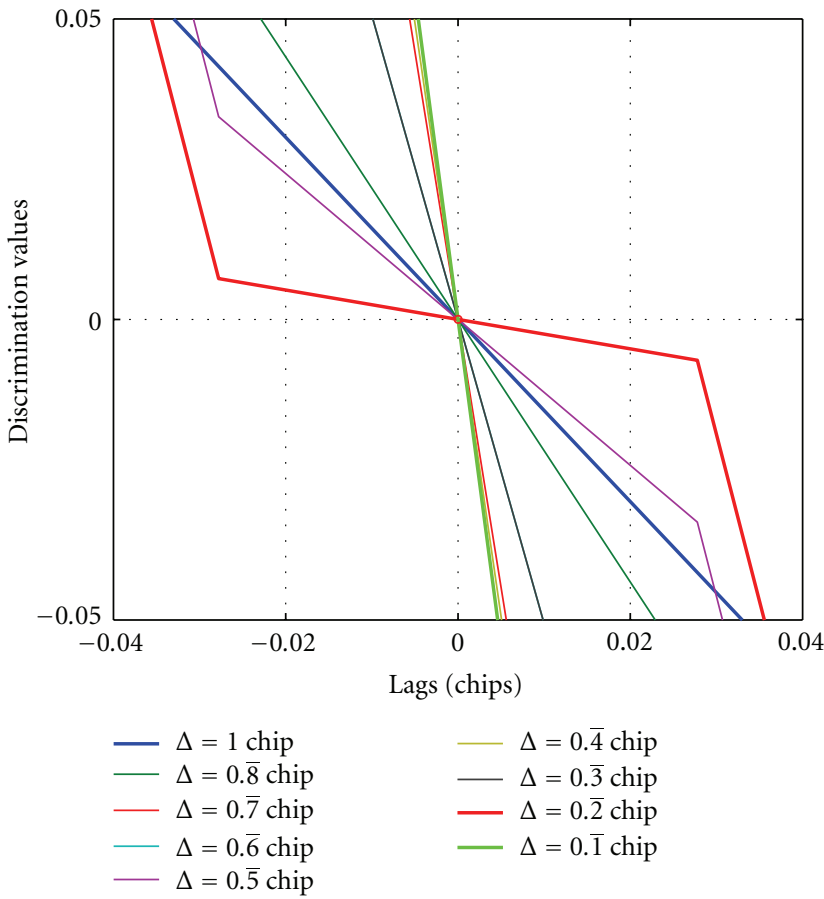

(b)

FIGURE 10: Theoretical coherent early-late discrimination function (a) and its zoom (b) varying the correlator spacing $(\Delta)$, considering only the pilot channel of a GPS L1C-like signal (TMBOC modulation, $\alpha=0$ ) and using Galileo E1 4 ms primary codes (PRN 1).

to pay is that longer integration times are needed to align the local code with the incoming signal.

Simulating the GPS L1C signal with the PRN 1 Weil codes for data and pilot channels, the discrimination function in Figure 7 is affected by a bias of $0.09 \mathrm{~m}$ (smaller than that of the Galileo case).

\section{S-Curve Analysis Varying the Receiver Setup}

As previously outlined, in addition to the code and modulation features, the actual impact of cross-correlations on the discrimination function also depends on the receiver setup. Several parameters and architectural choices, including the correlator type and spacing, can lead to discrimination functions with different shapes and slopes in the lock point, affecting the receiver performance. A complete analysis of the shape and the slope of this discrimination function with MBOC signals has then been performed varying the correlator spacing and considering the well-known coherent early-late discriminator.

4.1. Galileo E1 OS. Simulation results obtained using a Galileo E1 OS-like signal are presented in Figure 8, where theoretical S-curves are plotted for different arbitrary spacing values (multiple of 1/9 chip) and considering only the pilot channel. In this case, the S-curves have been obtained starting from the theoretical autocorrelation functions $R_{\mathrm{CBOC}_{+}}$ and $R_{\mathrm{CBOC}}$ computed on a single chip (previously shown in Figure 2) and neglecting the data/pilot cross-correlation effects $\left(R_{\mathrm{CBOC}_{+}}\right)$.

Observing the slope around the lock point in Figure 8(b), it is possible to notice that the steepest zero-crossing is obtained for $\Delta=0 . \overline{1}$ chip (green line). An important remark is that the trend of the slope is not directly related to the correlator spacing: a reduction of the spacing does not always lead to a steeper zero-crossing. The lowest slope, leading to the poorest receiver performance, is experienced using two spacings: $\Delta=0 . \overline{2}$ chip (purple line) and $\Delta=1 \mathrm{chip}$ (red line).

A detailed analysis on the changes of the S-curve slope has been performed varying the spacing with a tiny step in the range $(0,1]$ chip. The results are shown in Figure 9 for both data and pilot channels of a Galileo E1 OS-like signal.

It is easy to observe from Figure 9 that the S-curve slope can assume only a finite number of values and the slope variations occur at multiple of $1 / 6(0.1 \overline{6})$ chip spacing. The range of possible slopes is larger receiving only the pilot channel and the steepest slope is obtained with a spacing smaller than 1/6 chip. Another remark is that the obtained slope values near to the correct lock point assume different magnitudes, but always the same sign (negative): in this case, an inversion on the S-curve slope, leading to false locks, has not been noticed varying the early-late correlator spacing.

4.2. GPS L1C. Previous analyses have been repeated also using a GPS L1C-like signal. The shape of the S-curve for the pilot channel (TMBOC modulation) using arbitrary 


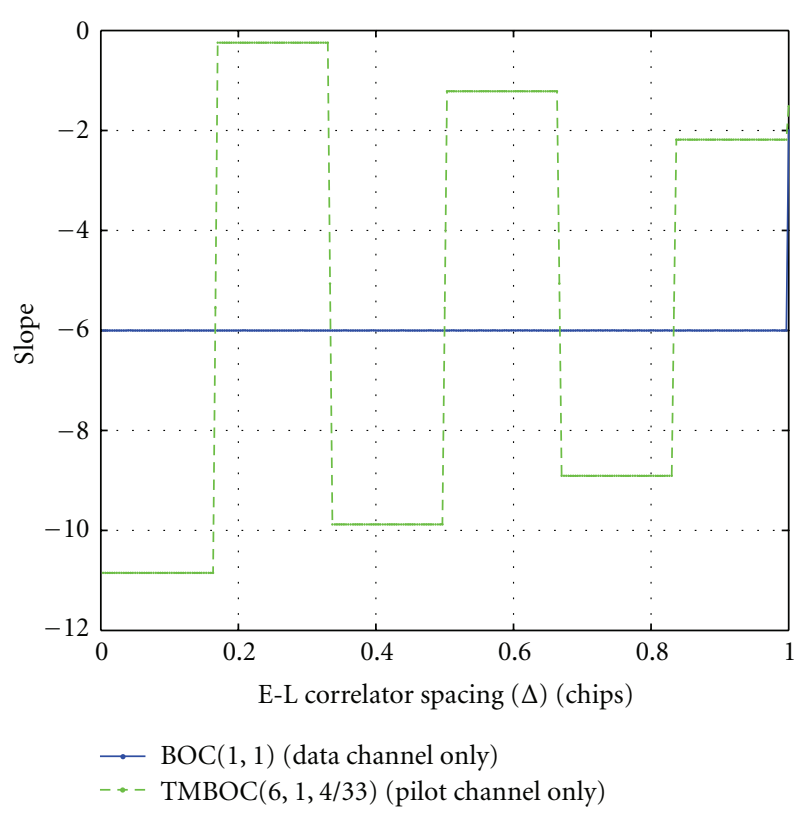

FIGURE 11: Theoretical S-curve slope varying the correlator spacing, considering the data and pilot channels of a GPS L1C-like signal (BOC and TMBOC modulations, $\alpha=0$ ) and using Galileo E1 $4 \mathrm{~ms}$ primary codes (PRN 1).

spacings is depicted in Figure 10. Comparing Figures 10 and 8 , it can be noticed that in this case, the worst slope is obtained only in one case ( $\Delta=0 . \overline{2}$ chip, red line). The spacing $\Delta=1$ chip (blue line) using the TMBOC leads to an intermediate slope between the worst case $(\Delta=0 . \overline{2}$ chip $)$ and the steepest slope $(\Delta=0 . \overline{1}$ chip).

A detailed analysis of the S-curve slope varying the spacing has been performed considering both data and pilot channels of GPS L1C, as reported in Figure 11. In this case, the slope for the L1C data channel is constant, due to the autocorrelation properties of the $\operatorname{BOC}(1,1)$ modulation.

On the other hand, the S-curve slope obtained using the pilot channel (TMBOC modulation) shows a similar behavior than the results with the Galileo pilot. In addition, as in the $\mathrm{CBOC}$ case, a variation on the early-late spacing does not lead to an inversion on the S-curve slope. These two cases are also compared in Figure 12, where small differences can be noticed.

The slopes in Figures 9, 11, and 12 have been computed assuming unitary autocorrelation functions. Taking into account also the relative power levels of data and pilot channels, it is demonstrated that the GPS L1C pilot channel leads to steeper discrimination functions than the Galileo E1 OS pilot channel (75\% of the total power versus $50 \%$ ). This does not prevent from drawing general conclusions about the relation between the receiver setup and the discrimination function. The correlator spacing impacts in a similar way on the shape and the slope of the S-curve using the pilot channels of the GPS L1C and Galileo E1 OS signals. In both cases, inappropriate choices of the spacing (e.g., $\Delta=0 . \overline{2}$ chip) can lead to a reduced slope, resulting

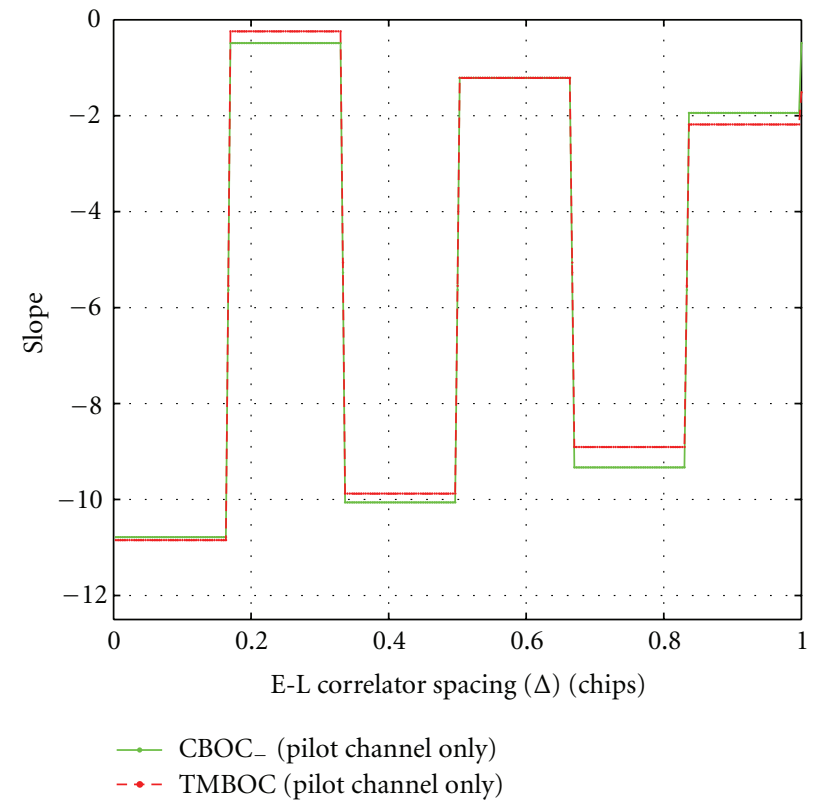

FIGURE 12: Comparison between theoretical slopes of the discrimination function varying the correlator spacing $(\Delta)$ and considering only the pilot channels of a GPS L1C-like signal (TMBOC modulation, $\alpha=0$ ) and a Galileo E1 OS-like signal (CBOC modulation, $\alpha=0$ ).

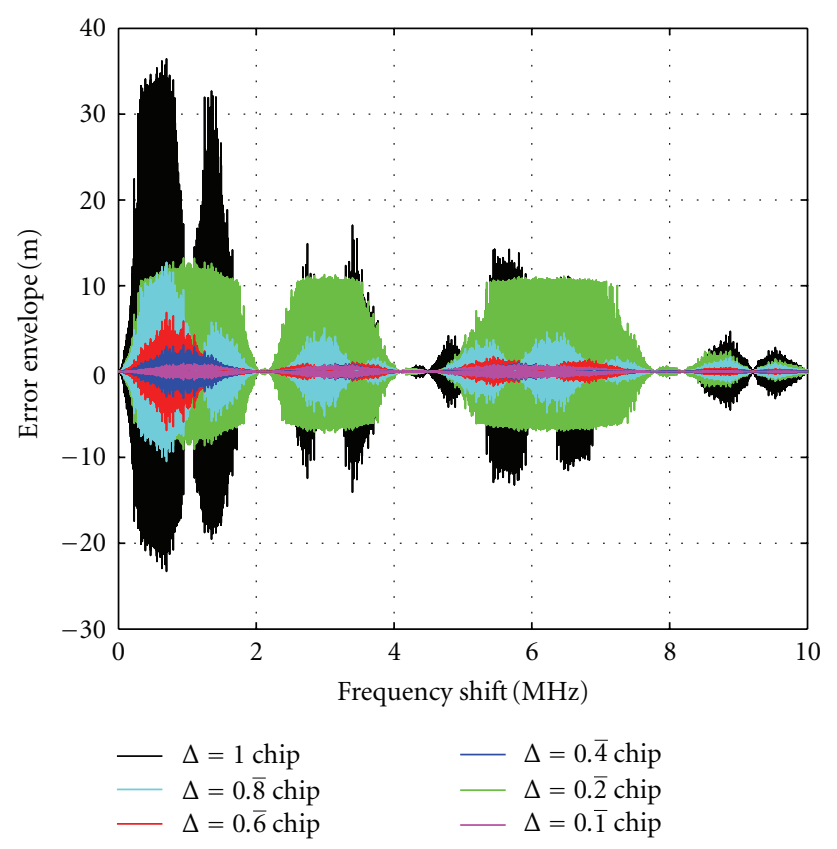

FIGURE 13: Interference Error Envelope comparison assuming different correlator spacings $(\Delta=1 \div 0 . \overline{1}$ chip) and receiving only the pilot channel of a Galileo E1 OS signal (CBOC modulation, $P R N$ 1) in presence of CW interference (carrier to interference power ratio equal to $0 \mathrm{~dB}$ ). 


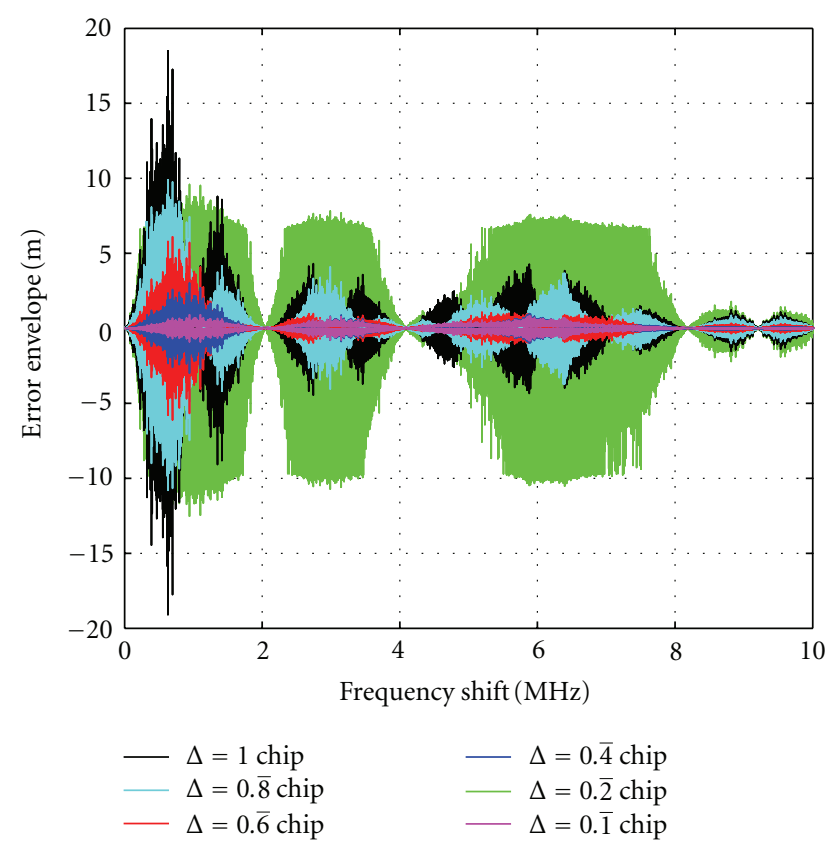

FIgURE 14: Interference Error Envelope comparison assuming different correlator spacings $(\Delta=1 \div 0 . \overline{1}$ chip) and receiving only the pilot channel of a GPS L1C-like signal (TMBOC modulation, using Galileo E1 PRN 1 primary code) in presence of CW interference (carrier to interference power ratio equal to $0 \mathrm{~dB}$ ).

in a discrimination function more vulnerable to code crosscorrelation distortions, as discussed in the following section.

\section{Simulation Results in Presence of an Interfering Signal}

The distortion induced by the cross-correlation of the channel not locally processed is not just threatening for the bias induced, but also the sensitivity to other error sources might increase. In this section, we show how the presence of an interfering source induces larger errors than expected. In order to compare the results, the interference error envelope (IEE) defined in $[7,13,14]$ is used. The IEE curves measure the correlation distortion versus specific interferer characteristics (e.g., the carrier frequency for a CW interferer); the corresponding interference running average (IRA) curves can be easily derived averaging the interference error envelopes.

IEE result for Galileo E1 OS and GPS L1C signals in presence of $\mathrm{CW}$ interference and are then presented in Figures 13 and 14 respectively. In detail, the IEEs have been computed simulating the reception of Galileo E1 OS and GPS L1C-like signals using only local pilot codes and varying the early-late spacing.

Observing the results in Figure 13 for the Galileo E1 OS signal, it is easy to notice that the worst case ranging error strongly depends on the chosen correlator spacing. All error envelopes are nearly symmetrical, except the two cases corresponding to the spacings $\Delta=1$ chip and $\Delta=0 . \overline{2}$ chip: they lead to a clear asymmetry and to worse errors.

This effect can be explained taking into account previous remarks about code features and receiver setup. As previously shown in Figure 8, both the spacings $\Delta=1$ chip and $\Delta=0 . \overline{2}$ chip lead to a low S-curve slope. This fact implies a noticeable vulnerability of the discriminator to possible distortions due to code cross-correlations and interference effects. In fact, assuming a low slope, the ranging errors in presence of an interfering signal result magnified. In addition, a noticeable asymmetry of the S-curve around the zero crossing (different slopes on each side) due to the data/pilot code crosscorrelations leads to different magnitudes for positive and negative errors, as in Figure 13 (black and green envelopes).

Similar results have been obtained simulating a GPS L1Clike pilot channel in presence of a CW interference (see Figure 14).

In this case, the asymmetry on the envelope can be noticed only for the spacing $\Delta=0 . \overline{2}$ chip: in fact, as previously observed in Figure 10, a low S-curve slope is obtained only using such a spacing.

In conclusion, it must be remarked that both using Galileo E1 OS and GPS L1C signals the code crosscorrelation distortion on the S-curve can be magnified by an inappropriate choice of the correlator spacings and it can lead to noticeable worsening in receiver performance in presence of an interfering signal. Such an effect can be noticed only in case of receiving a single channel (i.e., the pilot channel), whereas it is not present if the received signal is correlated with a coherent local replica including both data and pilot channels.

\section{Conclusions}

A comparative analysis of GPS L1C and Galileo E1 OS signals has been performed, pointing out how, when only the pilot channel is locally received in order to perform acquisition with long integration times, the residual cross-correlation due to the unprocessed channel cannot be neglected. Analytical and simulation results have been presented in order to demonstrate that in such a case the discrimination function can be affected by a bias around the lock point. The distortion can be noticed only in case of receiving a single channel (i.e., the pilot channel), whereas it is not present if the received signal is correlated with a coherent local replica including both data and pilot channels. It has also been shown how the distortion of the S-curve increases the sensitivity to other error sources, as for example to the presence of interfering signals. Moreover, the paper demonstrated that, in this case, inappropriate choices of the correlator spacing can lead to a discrimination function with reduced slope, thus enhancing the vulnerability of the receiver.

\section{References}

[1] M. Fantino, P. Mulassano, F. Dovis, and L. Lo Presti, "Performance of the proposed galileo CBOC modulation in heavy multipath environment," Wireless Personal Communications, vol. 44, no. 3, pp. 323-339, 2008. 
[2] Global Positioning System Wing Systems Engineering \& Integration, Interface Specification IS-GPS-800, Revision A. Navstar GPS Space Segment/User Segment L1C Interface, 2011, http://www.navcen.uscg.gov/pdf/gps/IS-GPS-800A_Final_08Jun10.pdf.

[3] European Union, European GNSS (Galileo) Open Service Signal In Space Interface Control Document, OS SIS ICD, Issue 1.1, 2010, http://ec.europa.eu/enterprise/policies/satnav/ galileo/open-service/index_en.htm.

[4] F. van Diggelen, Assisted GPS, GNSS, and SBAS, Artech House, 2009.

[5] D. Margaria, S. Savasta, F. Dovis, and B. Motella, "Codes cross-correlation impact on the interference vulnerability of Galileo E1 OS and GPS L1C signals," in Proceedings of the ION International Technical Meeting (ITM '10), pp. 1111-1121, San Diego, Calif, USA, January 2010.

[6] E. Rebeyrol, O. Julien, C. MacAbiau, L. Ries, A. Delatour, and L. Lestarquit, "Galileo civil signal modulations," GPS Solutions, vol. 11, no. 3, pp. 159-171, 2007.

[7] D. Margaria, S. Savasta, F. Dovis, and B. Motella, "Comparative interference vulnerability assessment of GPS TMBOC and Galileo CBOC signals," in Proceedings of the 22nd International Technical Meeting of the Satellite Division of the Institute of Navigation (ION GNSS '09), pp. 38-48, Savannah, Ga, USA, September 2009.

[8] J. W. Betz et al., "Enhancing the future of civil GPS—overview of the L1C signal," Inside GNSS, pp. 42-49, 2007.

[9] R. Gold, "Optimal binary sequences for spread spectrum multiplexing," IEEE Transactions on Information Theory, vol. 13, no. 4, pp. 619-621, 1967.

[10] P. Misra and P. Enge, Global Positioning System. Signal Measurements and Performance, Ganga-Jamuna, Lincoln, Mass, USA, 2nd edition, 2006.

[11] G. W. Hein, "MBOC: the new optimized spreading modulation recommended for Galileo L1 OS and GPS L1C," Inside GNSS, pp. 57-66, 2006.

[12] G. W. Hein, J. A. Avila-Rodriguez, and S. Wallner, "The Galileo code and others," Inside GNSS, pp. 62-74, 2006.

[13] B. Motella, S. Savasta, D. Margaria, and F. Dovis, "Method for assessing the interference impact on GNSS receivers," IEEE Transactions on Aerospace and Electronic Systems, vol. 47, no. 2, pp. 1416-1432, 2011.

[14] B. Motella, S. Savasta, D. Margaria, and F. Dovis, "A method to assess robustness of GPS C/A code in presence of CW interferences," Integrating Radio Positioning and Communications, vol. 2010, Article ID 294525, 8 pages, 2010. 

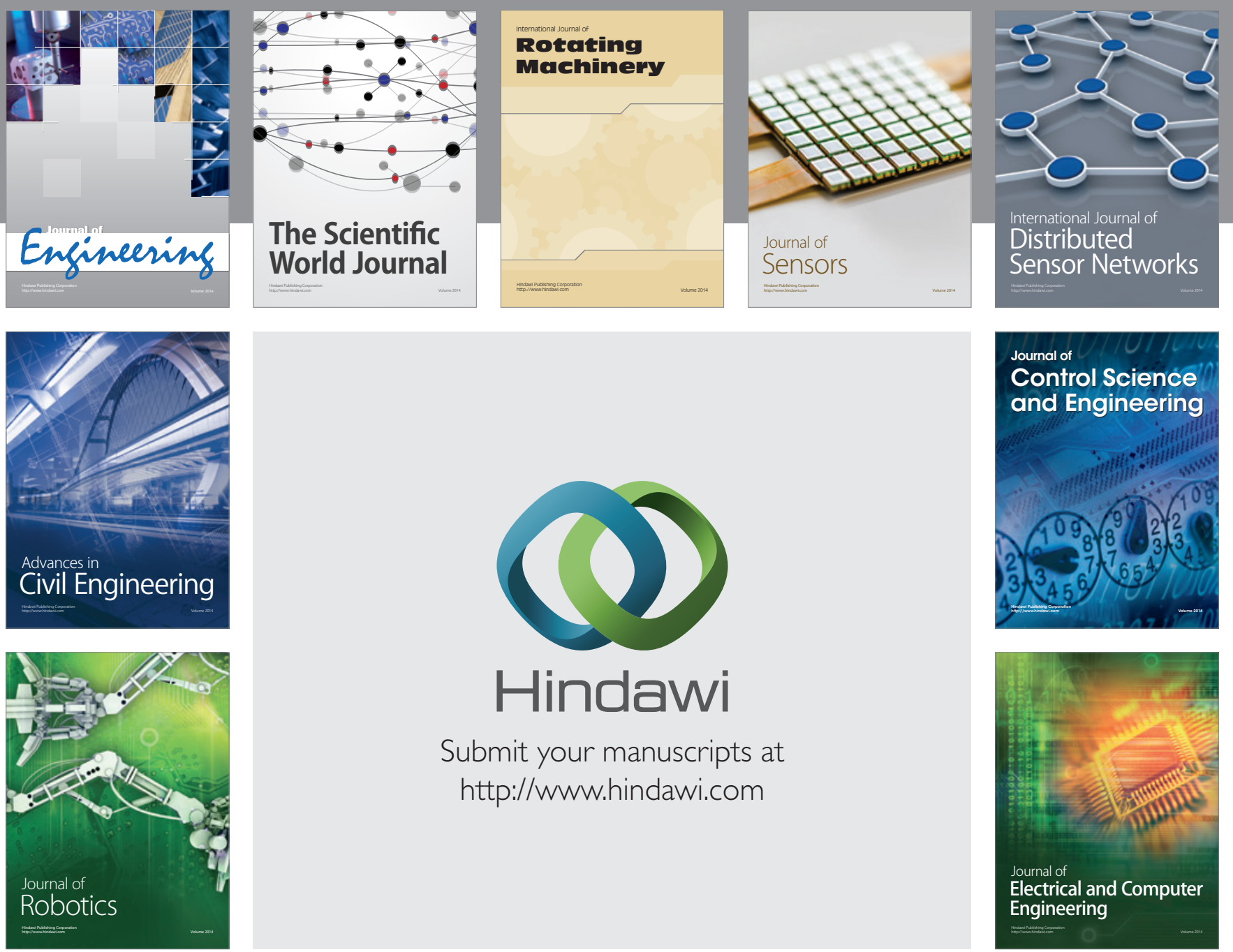

Submit your manuscripts at

http://www.hindawi.com
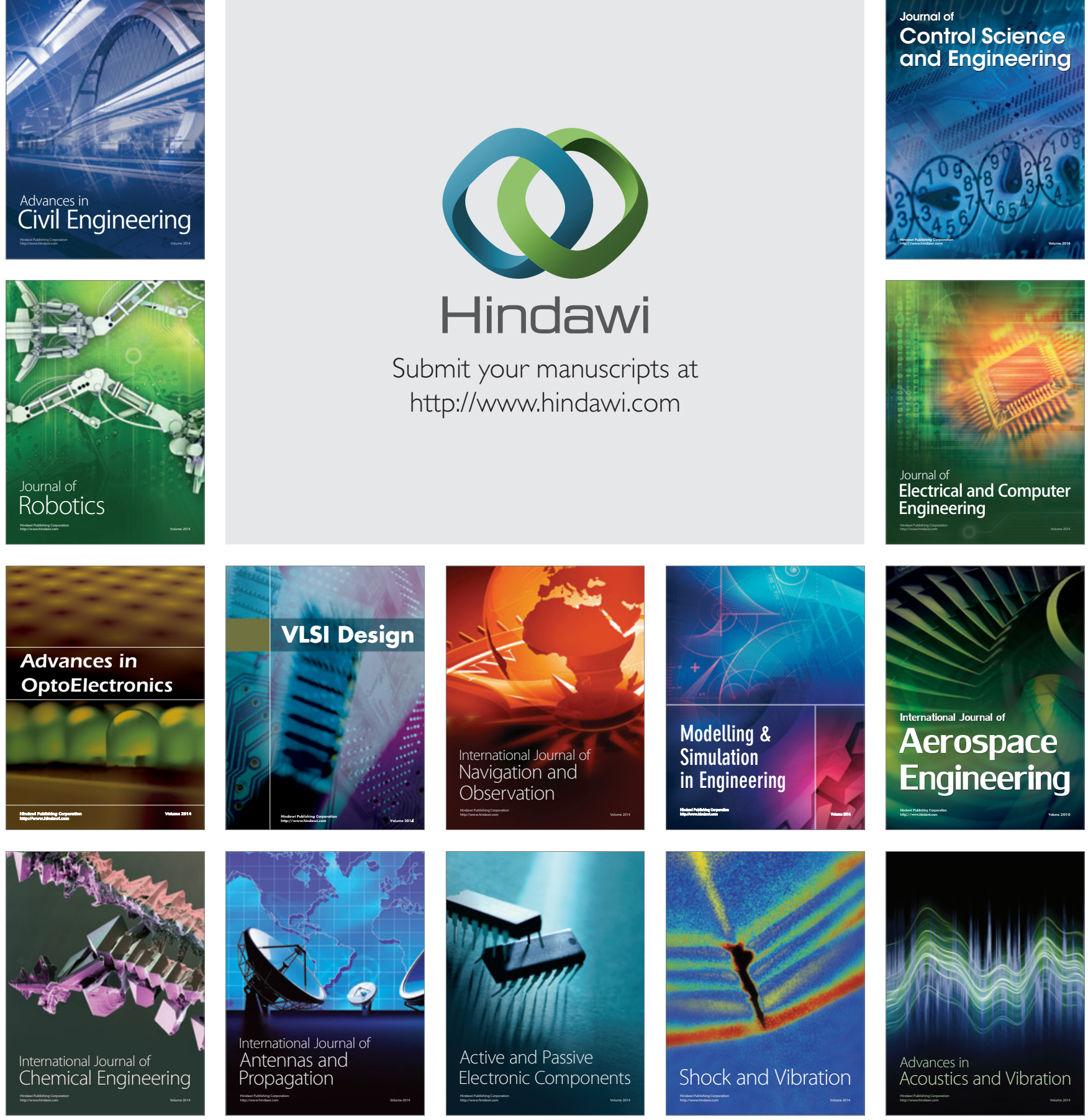